\title{
Colloidal Nanocrystals Embedded in Macrocrystals: Robustness, Photostability, and Color Purity
}

\author{
Tobias Otto, ${ }^{\dagger}$ Marcus Müller, ${ }^{\dagger}$ Paul Mundra, ${ }^{\dagger}$ Vladimir Lesnyak, ${ }^{\dagger}$ Hilmi Volkan Demir, ${ }^{\ddagger}, \S$
}

Nikolai Gaponik, ${ }^{* \dagger}$ and Alexander Eychmüller ${ }^{\dagger}$

${ }^{\dagger}$ Physical Chemistry/Electrochemistry, Technische Universität Dresden, Bergstr. 66b, 01062 Dresden, Germany

${ }^{\ddagger}$ Department of Physics, Department of Electrical and Electronics Engineering, and UNAM-Institute of Materials Science and Nanotechnology, Bilkent University, TR-06800, Ankara, Turkey

${ }^{\S}$ School of Electrical and Electronic Engineering and School of Physical and Mathematical Sciences, Nanyang Technological University, Singapore 639798, Singapore

Supporting Information

ABSTRACT: The incorporation of colloidal quantum dots (QDs) into ionic crystals of various salts $(\mathrm{NaCl}, \mathrm{KCl}, \mathrm{KBr}$, etc. $)$ is demonstrated. The resulting mixed crystals of various shapes and beautiful colors preserve the strong luminescence of the incorporated QDs. Moreover, the ionic salts appear to be very tight matrices, ensuring the protection of the QDs from the environment and as a result providing them with extraordinary high photo- and chemical stability. A prototype of a white light-emitting diode (WLED) with a color conversion layer consisting of this kind of mixed crystals is demonstrated. These materials may also find applications in nonlinear optics and as luminescence standards.

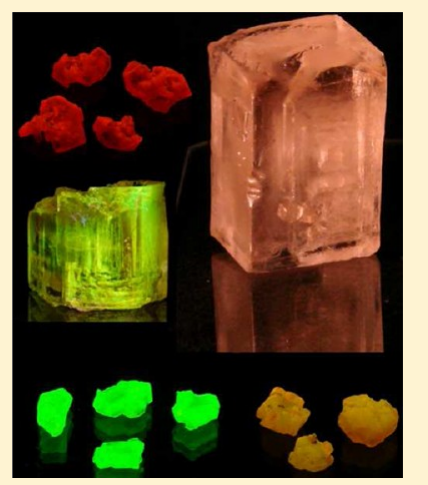

KEYWORDS: Quantum dots, nanocrystals, mixed crystals, composite, color conversion, white LED

hemically synthesized semiconductor quantum dots (QDs) attract more and more interest as components

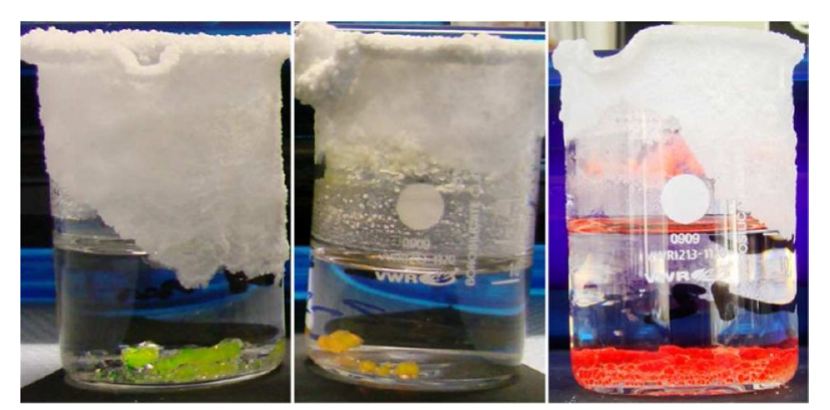

Figure 1. Final stage of the $\mathrm{NaCl}$ crystallization in the presence of CdTe QDs of various sizes. Mixed crystals formed on the bottom of the glass appear colored due to embedded QDs.

of modern optoelectronic and photovoltaic devices. ${ }^{1}$ Lightemitting diode (LED) operation by direct current injection into QD layers has been reported for about 18 years. ${ }^{2,3}$ Many improvements regarding efficiency, ${ }^{4}$ simplification of fabrication, ${ }^{5}$ new architectures, ${ }^{6-9}$ extension of spectral range, ${ }^{10-12}$ environmental friendliness, ${ }^{13}$ and so forth have been achieved since that time. One of the most common challenges is to reach a reasonable stability of the QDs under conditions of direct injection of charge carriers, as well as their photostability. Protecting shells should be thick and defect-free to provide isolation from the environment but thin enough to facilitate charge transport. A proper balance between these two issues allows for some improvement, but the resulting devices are still less efficient than the quickly emerging organic or polymer LEDs (OLEDs or PLEDs, respectively). ${ }^{9}$ The question arises why QD-based LEDs are still attractive? The main reasons lay in the following unique properties of QDs: narrow and tunable photoluminescence (PL) spectra as well as thermal stability inherent to inorganic materials. Indeed, the narrow photoluminescence spectra provide high color purity which is necessary for the precise design of high quality lighting. ${ }^{14,15}$ This level of color purity is not achieved with the relatively broad emission of organic dyes. The tunability of the QD emission spectra allows one to obtain PL maxima from the UVblue to the near-infrared. In its own turn the long-term stability above $90{ }^{\circ} \mathrm{C}$ is a critical issue for applications of LEDs; that is, in the automotive industry as elements of modern dash-boards or signal lighting. ${ }^{16}$

Presently, solid state lighting (SSL), that is, lighting based on LEDs, attracts enormous attention. ${ }^{17}$ In energy and cost

\footnotetext{
Received: July 24, 2012

Revised: September 11, 2012

Published: September 14, 2012
} 

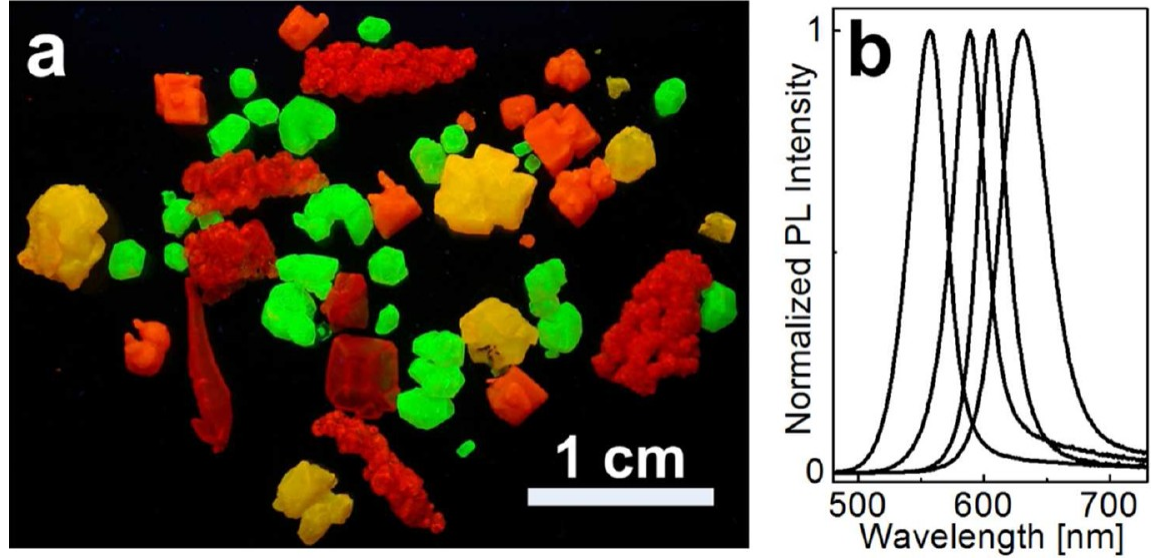

Figure 2. (a) A true color image of several NaCl-based mixed crystals containing CdTe QDs of different sizes and thus emission colors. The image is taken under excitation by a $365 \mathrm{~nm}$ UV lamp. (b) PL spectra of various mixed crystals from panel a.

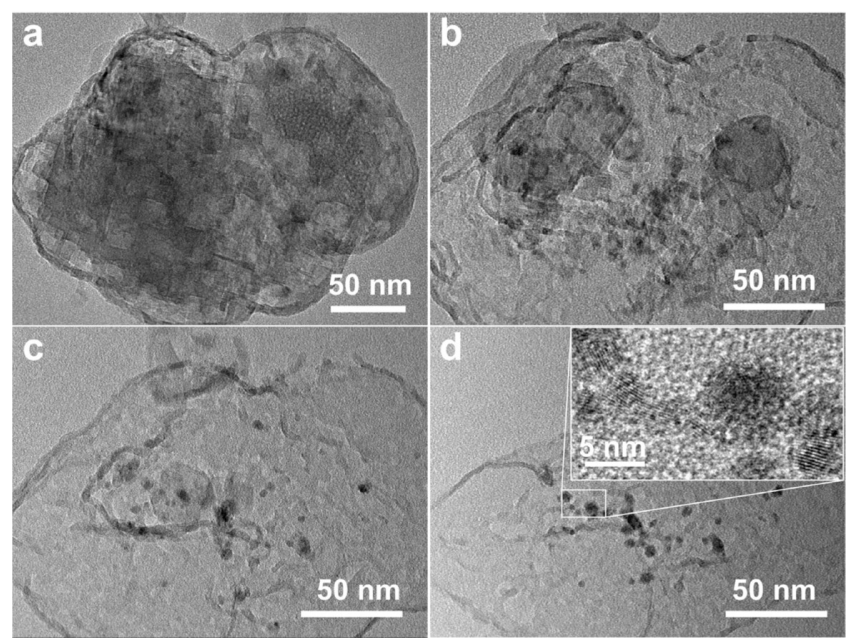

Figure 3. Representative TEM (a-d) and high resolution TEM (inset) images of a mixed crystal sample $(\mathrm{NaCl}+\mathrm{CdTe} \mathrm{QDs})$. Images have been taken from the very same part of the sample. The evolution of the appearance of the sample (from a to d) is due to melting of the ionic crystal under the electron beam.

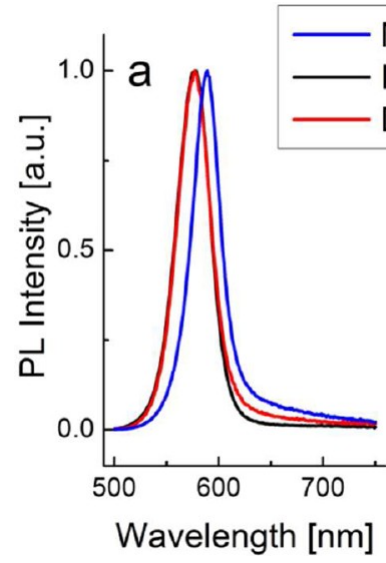

Mixed crystal

Initial NCs solution

Dissolved crystal

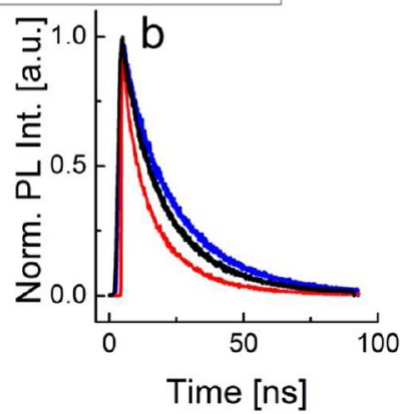

Figure 4. Steady state photoluminescence spectra $\left(\lambda_{\mathrm{ex}}=450 \mathrm{~nm}\right)(\mathrm{a})$ and time-resolved $\left(\lambda_{\mathrm{ex}}=470 \mathrm{~nm}\right)$ decay traces (b) of an initial aqueous solution of CdTe QDs (black), mixed CdTe QDs- $\mathrm{NaCl}$ crystals (blue) and of the aqueous solution obtained after dissolving of the mixed crystal (red).

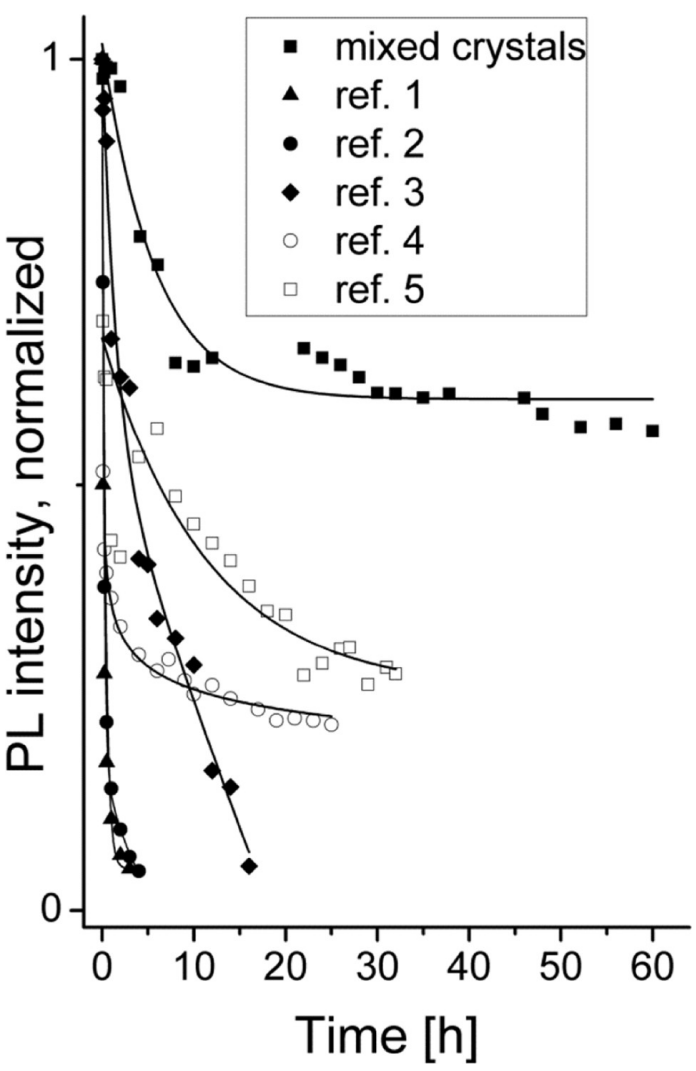

Figure 5. Evolution of the integral PL intensity of mixed CdTe QDs$\mathrm{NaCl}$ crystals and several reference samples containing the same QDs in different matrices or mixtures under ca. $1.0 \mathrm{~W} / \mathrm{cm}^{2}$ light intensity generated in the focus of a $1000 \mathrm{~W}$ xenon lamp with a luminance of ca. $1800 \mathrm{~cd} / \mathrm{cm}^{2}$. Reference samples are: mixtures of CdTe QD powders with PMMA-powder (ref 1), $\mathrm{NaCl}$ powder (ref 2), and glass powder (ref 3) as well as CdTe QDs embedded in bulk polymers: PMMA/PS (ref 4) and PS (ref 5). Solid lines are solely provided as a guide to the eye.

efficiency this type of lighting outperforms the traditional incandescent bulbs even with today's technologies. Moreover, according to even very conservative projections SSL already carries the potential for overcoming fluorescent and high intensity discharge light sources in the next decade. ${ }^{17}$ Typical SSL devices exist in two possible architectures. ${ }^{17,18}$ One is based on the simultaneous use of blue, green, and red emitting 


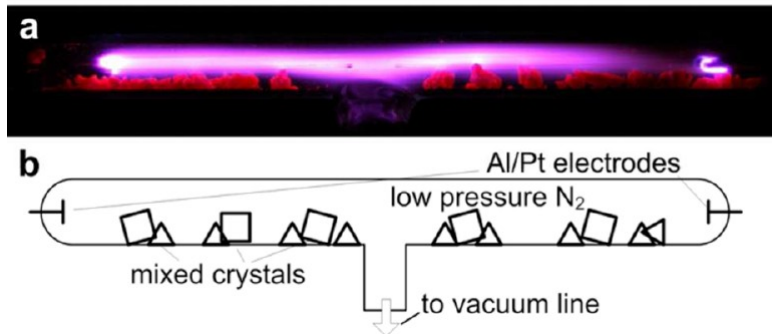

Figure 6. True color image (a) and schematics (b) of a homemade $\mathrm{N}_{2}$ gas discharge lamp containing red emitting $(657 \mathrm{~nm})$ mixed CdTe QDs- $\mathrm{NaCl}$ crystals.

LEDs. The other architecture includes a UV-blue emitting LED-chip combined with color converting phosphors.

By about 12 years ago, the first reports on QD containing LEDs utilizing the principles of color conversion had appeared. ${ }^{19}$ Here no direct charge carrier injection is involved, and therefore, degradation due to electrochemical corrosion or electrodiffusion of atoms is not inherent. Moreover, the requirements as to the architecture of the $\mathrm{QD}$-containing part of the device are simplified to a large extent (there is no necessity to form perfect monolayers or to design defect-free, multilayered superstructures of QDs and semiconducting polymers with nanometer precision). A good choice of material combinations includes that of a most efficient LED platform (usually a UV or UV-blue emitting single crystal III-V LED) and a light conversion layer with the highest possible photoluminescence quantum efficiency, a suitable refractive index, good photostability, and a desired color. It is not surprising that among the QD-based devices, namely, these kinds of nano-LEDs, based on color conversion principles presently carry the highest application potential. Indeed, a 40 in. flat screen display prototype with brilliant full color characteristics determined by a QD-backlighting module was presented by Samsung researchers recently. ${ }^{20}$ Other examples include the demonstration of efficient color control ${ }^{15,21}$ and the utilization of less toxic QDs. ${ }^{22}$ However, a full protection of QDs which provides high photostability and processability while not simultaneously deteriorating their photoluminescence is still a challenging task. For instance, the rigid chemical conditions used in the polymerization of silicone or sol-gel syntheses of inorganic oxides which are commonly used materials for the encapsulation of light emitting entities deteriorate the PL of the embedded QDs.

In this paper, we report on the fabrication of robustly and strongly emitting processable composites based on colloidal quantum dots incorporated into conventional salt crystals, that is, $\mathrm{NaCl}, \mathrm{KCl}, \mathrm{KBr}$, and so forth. In contrast to salts doped with semiconductor particles by the Czochralski approach, ${ }^{23}$ the mixed crystals presented here are grown at ambient temperatures by the crystallization from saturated aqueous solutions of the respective salts additionally containing colloidally prepared, strongly photoluminescing QDs. These mixed crystals display an extremely robust optical performance; their photoluminescence and absorption properties are determined by the type,
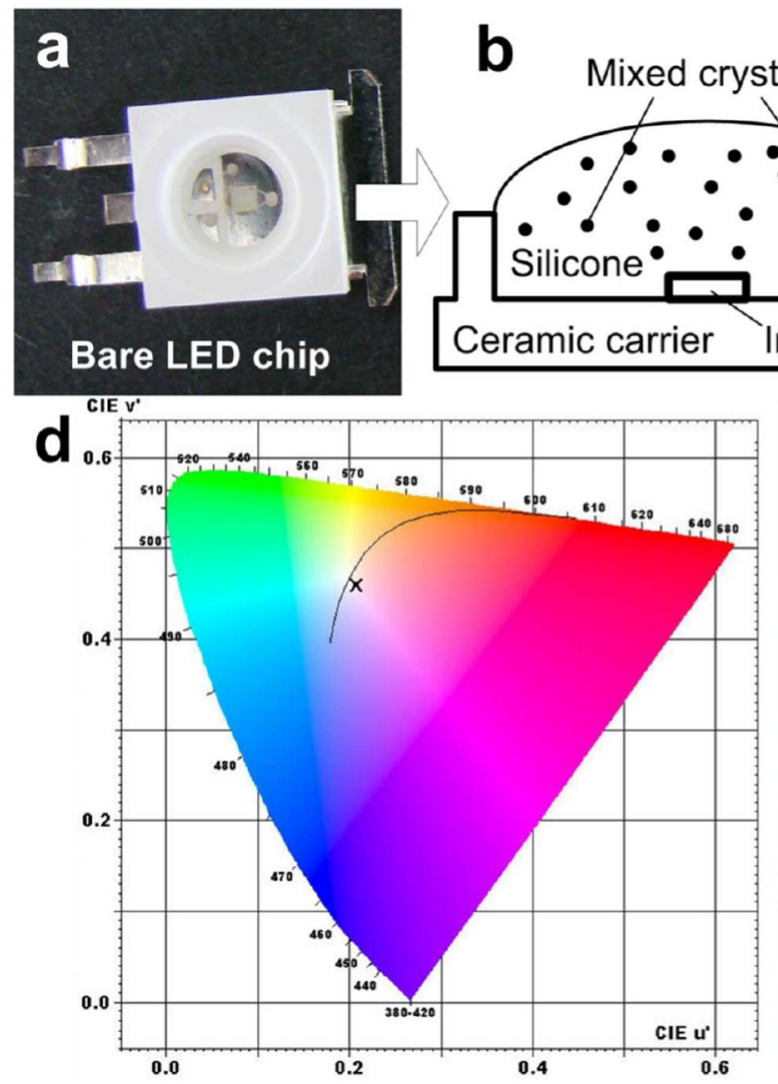
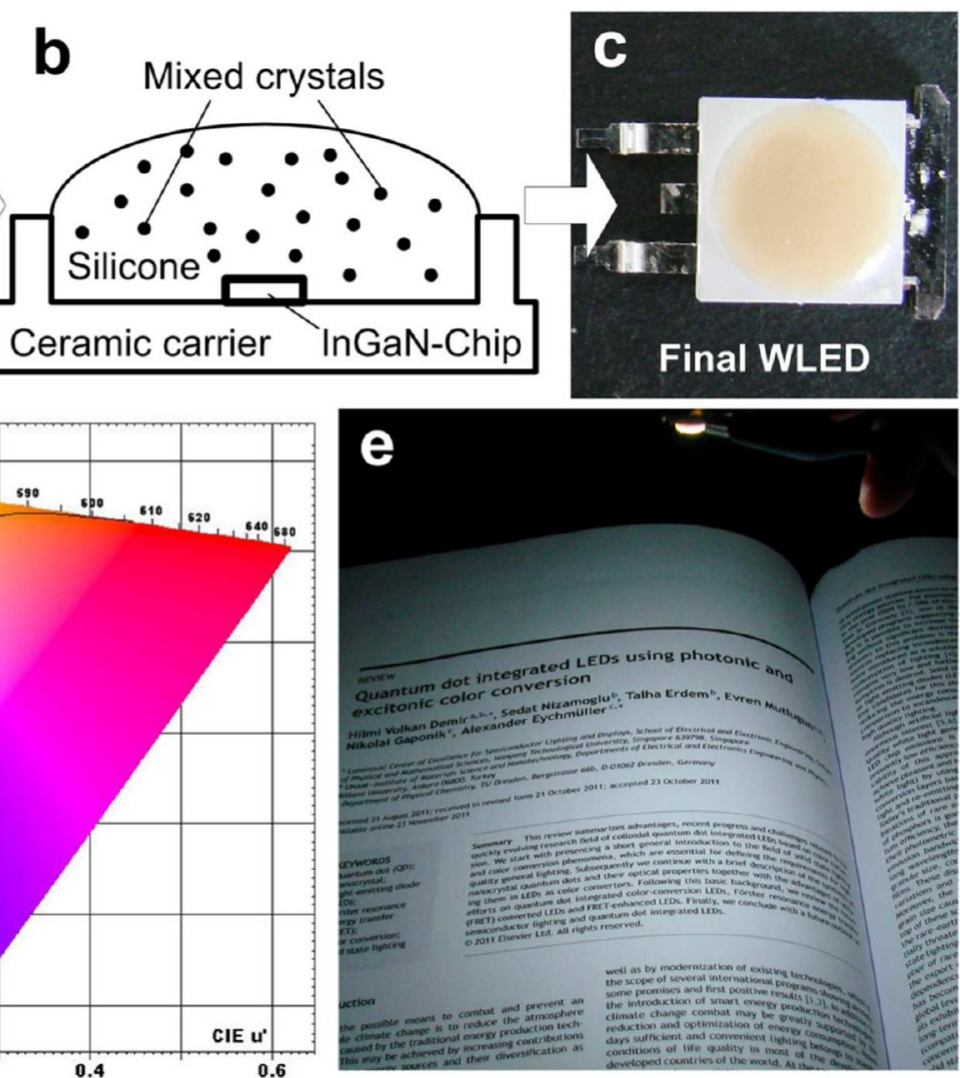

Figure 7. Bare blue emitting $1 \mathrm{~W}$ InGaN LED chip (a), schematics of its hybridization with mixed crystals embedded in silicone (b), and the resulting white LED (c). Color coordinates of the WLED are shown as a cross in the CIE 1976 diagram (d). The color temperature of the device as derived from the nearest point in the Planckian locus is around $6500 \mathrm{~K}$. A title page reprinted with permission from ref 15 illuminated by the WLED (e). 
size and size distribution of the nanocrystals used. These crystals may be further processed by mixing them with epoxy resin, silicone, poly(methyl methacrylate) (PMMA), or other typical matrices without any deterioration of their optical properties which opens perspectives for their applications in optoelectronics and photonics, for example, as color convertors, optical gain media, and as elements of optical circuits.

Experimental Section. Chemicals and Apparatus. All chemicals used were of analytical grade or of the highest purity available. For all solutions, Milli-Q water (Millipore) was used as a solvent. The $\mathrm{Al}_{2} \mathrm{Te}_{3}$ lumps used for the generation of $\mathrm{H}_{2} \mathrm{Te}$ were purchased from CERAC Inc.

UV-vis absorption spectra were recorded using a Cary 50 spectrophotometer (Varian). Photoluminescence (PL) measurements were performed at room temperature using a FluoroMax-4 spectrofluorimeter (Horiba Jobin Yvon). The PL quantum efficiency ( $\mathrm{QE}$ ) of the CdTe nanocrystals was measured at room temperature as described in ref 24 by a comparison with Rhodamine 6G and Rhodamine 101 (both Radiant Dyes Laser) in ethanol (Uvasol, Merck), assuming their PL QE as 95\% and 96\%, respectively. The photochemical treatment of the crystal samples was done with a $1000 \mathrm{~W}$ xenon short arc lamp with a water filter for cutting off the nearinfrared (NIR) part of the spectrum. The luminance of the light was approximately $1800 \mathrm{~cd} / \mathrm{cm}^{2}$, measured with a MINOLTA Chroma Meter CS-100A. Samples for transmission electron microscopy (TEM) were prepared by positioning samples onto copper grids coated with a thin Formvar-carbon film. TEM imaging was carried out on a Tecnai T20 microscope operating at $200 \mathrm{kV}$ (FEI).

Synthesis of Nanocrystals. The synthesis was performed according to ref 25 . In a typical synthesis, $2.305 \mathrm{~g}(5.5 \mathrm{mmol})$ of $\mathrm{Cd}\left(\mathrm{ClO}_{4}\right)_{2} \cdot 6 \mathrm{H}_{2} \mathrm{O}$ was dissolved in $250 \mathrm{~mL}$ of water, and $7.15 \mathrm{mmol}$ of the thiol stabilizing agent was added dropwise under stirring, followed by an adjustment of the $\mathrm{pH}$ to 12 using a $1 \mathrm{M}$ solution of $\mathrm{NaOH}$. The solution was placed in a threenecked flask and deaerated with argon for $30 \mathrm{~min}$. The $\mathrm{H}_{2} \mathrm{Te}$ gas was generated by the reaction of $0.4 \mathrm{~g}(0.916 \mathrm{mmol})$ of $\mathrm{Al}_{2} \mathrm{Te}_{3}$ lumps with $10 \mathrm{~mL}$ of $0.5 \mathrm{M} \mathrm{H}_{2} \mathrm{SO}_{4}$ solution and passed through the solution with a slow argon flow under stirring. At this stage, CdTe nanocrystal precursors were formed. Further growth of the QDs proceeded under open air conditions upon refluxing at $100{ }^{\circ} \mathrm{C}$ with a condenser attached. Nanocrystals obtained by this well-established approach possess PL quantum efficiencies in the range of $40-60 \%{ }^{24}$ The obtained QDs were used for mixed crystallization from their crude (as prepared) solutions as these solutions show relatively high colloidal stability in concentrated salt media.

Synthesis of Mixed Crystals. The preparation of the crystals was performed by mixing $25 \mathrm{~mL}$ of a saturated salt solution $(\mathrm{NaCl}, \mathrm{KBr}$, or $\mathrm{KCl})$ with $5 \mathrm{~mL}$ of a $\mathrm{QD}$ solution in a $30 \mathrm{~mL}$ beaker. Protected from dust the solutions were stored under ambient conditions. For $\mathrm{NaCl}$ and $\mathrm{KCl}$, saturated solutions at room temperature were used. $\mathrm{KBr}$ solution could be used for crystallization at room or at an elevated temperature, since its solubility rises by a factor of 4 in going from 20 to $100{ }^{\circ} \mathrm{C} .{ }^{26} \mathrm{~A}$ higher solubility provides higher concentrations of saturated salts and as a result higher crystallization rates by cooling the solution down to room temperature. The crystallization was complete when the parental solution turned colorless. The crystals formed were removed from the parental solution, rinsed with cold water and dried. Although the influence of the colloidal species on the growth and structure of ionic crystals is expected, we did not observe any remarkable effects in our experiments. Most probably, the low loading values of the QDs into the crystals (below 1\%) are considered to be responsible for this observation.

Preparation of Mixed Crystal-Silicone Composites. For this, first the two components of the silicone precursor (QSil218, ACC Silicones) were mixed at a ratio of 10:1 (v/v). For the incorporation of the mixed crystals, they were placed in the silicone precursor mixture and cured in air at $80{ }^{\circ} \mathrm{C}$ for $2 \mathrm{~h}$. For the preparation of the LEDs, the crystals were milled to a fine powder. Then, $\sim 30 \mu \mathrm{L}$ of silicone precursor mixture was placed on the LED, and the desired amount of powder was subsequently added. After the dispersion of the powder within the silicone, the mixture was cured in a similar manner.

Results and Discussion. As described in the Experimental Section, the typical procedure for the preparation of mixed crystals is relatively simple and is adapted from well-known crystallization approaches which are described in the professional as well as in popular literature. ${ }^{27-29}$ Water-soluble QDs are mixed with saturated solutions of the corresponding salts and allowed to stay for periods of several days up to several weeks during which the slow evaporation of water, oversaturation, and consequently, crystallization take place. Alternatively, crystallization is possible by the cooling of hot saturated salt solutions. However, the resulting large crystallization rates lead to low nanocrystal loading. Therefore, all data presented below have been obtained following crystallization at room temperature. As seen from the images in Figure 1, the microcrystalline phase forming around the edges of the beakers is purely white, that is, QD free, while the macrocrystals formed on the bottom of the glass are remarkably colored due to the incorporation of QDs. Before further characterization, these mixed crystals were taken out of the beaker and rinsed with cold water to remove from the surface QDs or any byproducts of the synthesis precipitated or aggregated. We note that, in cases where the surface QD concentration was very high or the QDs had relatively low stability in salt media, some precipitation of QD aggregates may occur on the bottom of the beaker. To avoid unnecessary losses of QDs, the concentration ratio of the components in the solution for the mixed crystallization should experimentally be optimized for each batch of the QDs. The results reported below are obtained from mixed crystals crystallized via optimized routes.

All examples of the mixed crystals shown in the present paper originate from $\mathrm{NaCl}$-based materials. Examples of $\mathrm{KCl}, \mathrm{KBr}$, and $\mathrm{K}-\mathrm{Na}$ tartrate based mixed crystals are presented in Figure SI1 (see Supporting Information).

The main challenge in the preparation of mixed crystals is to achieve reasonably long-term stability of the colloidal QDs in the presence of concentrated salt solutions. Indeed, premature coagulation would result in precipitation of $\mathrm{QD}$ aggregates and their removal from the crystallization process. Thus, the QDs directly synthesized in water in the presence of short chain thiols are the most suitable for mixed crystallization. The necessary stabilization may be achieved, for example, in the presence of thioglycolic or mercaptopropionic acids. More details on the suitable stabilization of thiol-capped QDs may be found in the corresponding literature. ${ }^{30,31}$ It is noted, however, that also QDs synthesized in organic media by hot-injection approaches $^{32}$ (e.g., CdSe based core-shell QDs) may be embedded in macrocrystals. For this, two main strategies were preliminarily validated in the present work: (i) utilization of 
macrocrystals of organic substances and (ii) phase transfer of QDs from nonpolar solvents to water and subsequent crystallization as discussed above. Unfortunately, the first method delivers only relatively unstable organic-based crystals which cannot be considered as robust and stable matrices for QDs. In its own turn, the application of the second method is limited by the relatively high tendency of phase-transferred QDs to coagulation in strong ionic media. Despite this drawback, we have achieved a success by forming mixed $\mathrm{NaCl}$ crystals embedded with $\mathrm{CdSe} / \mathrm{CdS}$ QDs prepared via hot-injection. The results, however, are of preliminary character since the approach developed still needs optimization from the point of view of versatility and reproducibility (see Supporting Information, Figures SI2 and SI3 for more details). As this approach differs from the present one, the results will be published in a separate manuscript.

Figure 2 shows typical images of mixed $\mathrm{NaCl}$ based crystals containing CdTe QDs under UV-lamp excitation and their corresponding PL spectra. As is seen from the figure, the crystals exhibit the typical shapes and sizes of the corresponding salt materials and simultaneously show uniformly distributed emission from the incorporated CdTe QDs. The PL spectra of the crystals cover a spectral region ranging from ca. 550 to ca. $630 \mathrm{~nm}$.

TEM characterization of the salt crystals is complicated because the mixed crystal samples melt upon electron beam exposure. Figure 3 presents a series of images taken from the same small piece of a $\mathrm{NaCl}$ crystal containing CdTe QDs. As seen from the images, although separate QDs are not visible in the bulk crystal (Figure 3a), they may very well be recognized after melting has started (Figure $3 b-d$ ). Separate crystalline QDs are seen in the high-resolution TEM image of the melt (inset to Figure 3d). It was also observed that not aggregated but well-separated QDs dominated in the crystals (see, e.g., Figure $3 b, c)$. Partial aggregation as seen in Figure 3d occurs mainly during the melting. Based on these data we can suggest a probable mechanism of crystallization of the mixed crystals. Upon injection of QDs into the saturated salt solution individual nanoparticles play the role of seeds for the crystallization of the salt and are immediately "wrapped" by small crystallites of the respective salt. This salt shell protects the QDs from aggregation, and the small crystallites can merge and grow further finally yielding macrocrystals. We note that separation of the individual QDs in the matrix is very important for maintaining their original PL. As will be shown below, this is in good agreement with the PL characterization of the samples.

As seen from Figure 4, to a large extent the PL spectral characteristics of the mixed crystals resemble those of the QDs in solution. A red shift of the PL maximum is observed in the crystals and may be explained by changes of the dielectric constant of the surrounding media as well as by reabsorption of the blue part of the spectrum in optically dense samples. Energy transfer between the well-separated QDs in the composite is less probable. Indeed, no sufficient difference in the PL life times was observed between mixed crystal samples and the QDs in aqueous solution (Figure $4 \mathrm{~b}$ ). This is also taken as a direct evidence for the maintaining or even a slight improvement of the initial PL quantum yield of the embedded QDs. The shorter PL lifetime of the dissolved crystal sample is attributed to a partial aggregation of the QDs in the diluted ionic salt solution. Such a kind of lifetime shortening due to nonradiative recombination of excitons is commonly observed in QD aggregates. ${ }^{33,34}$
It is expected that the ionic salt provides an extraordinarily tight matrix for the embedded QDs. Indeed, it is very improbable, that ambient oxygen could penetrate through the salt to the encapsulated QDs. Thus, being protected from the environment, the QDs should offer high photostability. To perform photostability tests we mounted the mixed macrocrystal samples in the focus of a $1000 \mathrm{~W}$ xenon lamp equipped with a water filter to cut off the NIR part of the spectrum. The light intensity in the focus on the sample was approximately $1.0 \mathrm{~W} / \mathrm{cm}^{2}$. As reference samples we used CdTe QDs embedded in polymers (polystyrene and a mixture of polystyrene (PS)/poly(methylmetacrylate) (PMMA)), mixtures of $\mathrm{QD}$ powder with $\mathrm{NaCl}$ crystals powder, with glass powder and with PMMA powder (see the Supporting Information for details). All of the reference samples were carefully prepared to achieve comparable optical densities with the mixed crystal sample. To monitor the stability, the PL spectra of the samples were measured in the course of the phototreatment. As seen from Figure 5, the harsh conditions of the illumination used in our tests are indeed harmful to all of the reference samples. Even the CdTe QDs embedded in such common bulk polymer matrices as PMMA and PS lost more than a half of their initial PL intensity after the first $10 \mathrm{~h}$ of illumination. Unprotected QD mixtures with various powders totally degraded on time scales of minutes to several hours. Nevertheless, the mixed crystal sample showed a remarkable stability of the PL for more than $60 \mathrm{~h}$. The initial $30-40 \%$ drop of the emission intensity may be assigned to degradation processes involving water and oxygen entrapped in the mixed crystals during the crystallization procedure, for example, as inclusions in hydrate shells around the colloidal nanocrystals. Degradation of the QDs associated with the surface of the mixed crystal may also contribute to this initial emission drop. It should be stressed that we observed only changes of the integral PL intensity. The shape and position of the PL spectra were not affected by this intensive phototreatment. Some irregular changes of the PL intensity observed in the data may be explained by slight temperature deviations during the phototreatment and the measurements.

The mixed crystals were also shown to be stable for long times under conditions of excitation in a gas discharge lamp. For this experiment a gas discharge lamp was fabricated from a T-shape glass tube with two $\mathrm{Al} / \mathrm{Pt}$ electrodes melted in on opposite sides of the tube, as is schematically shown in Figure 6. The tube was filled with mixed crystals, evacuated to 6.7 mbar. Discharge in the remaining $\mathrm{N}_{2}$ (from air) was initiated by applying of $2.5 \mathrm{kV}$ (working current $1 \mathrm{~mA}$ ). Red emitting mixed crystals with a PL maximum at $657 \mathrm{~nm}$ exited by the $\mathrm{N}_{2}$ discharge are clearly seen in Figure 6a. This experiment was a first step toward the demonstration of the applicability of the mixed crystals for color conversion purposes.

To be used as color converters in solid state lighting devices, the QDs typically should be compatible with a silicone matrix which is commonly used for the hybridization of the color converter onto the LED platform. ${ }^{35}$ Most of the QDs are not compatible with silicone monomer solutions; moreover, the polymerization of typical silicones proceeds under rigid chemical conditions leading to complete or partial quenching of the QD PL. ${ }^{35}$ To overcome these limitations, the QD surfaces can be modified with silica. ${ }^{22}$ However, these $\mathrm{SiO}_{2}$ modified QDs, though being better compatible with silicone, exhibit a reduced PL efficiency. ${ }^{22}$ The mixed crystals obtained 
in this work can be easily milled, and the resulting powder can be added in desired amounts and color ratios to silicone polymerization solutions. By doing so, no degradation of the mixed crystals and their PL has been observed. Composites containing mixed crystal powders appear milky due to scattering and retain the emission colors corresponding to the initial QDs (see the Supporting Information, Figure SI4). Composites of this sort being deposited on commercially available $1 \mathrm{~W}$ blue InGaN LED chips allow to fabricate a proof of concept white light-emitting device (WLED) whose illuminating capacity as well as color coordinates are shown in Figure 7. We note that an optimization of this kind of devices, that is, achieving desired color coordinates, low color temperature (warm light), high color rendering index, control of scattering, thermostability, and so forth demand additional intensive investigations including modeling, optimization of design, and fabrication procedures and finally the application of the relevant characterization techniques which will be the topic of a forthcoming paper.

Conclusions. In summary, we have demonstrated a relatively simple and versatile approach allowing the incorporation of strongly photoluminescing colloidal QDs into various inorganic salt crystals. The high stability of the QDs inside the salt matrix, the processing into composites with silicone, and a proof of concept application of the mixed crystals to LED color conversion are demonstrated. These mixed crystals may find also applications in nonlinear optics (e.g., as gain media) and as fluorescence standards.

\section{ASSOCIATED CONTENT}

\section{(S Supporting Information}

Images of mixed crystals based on $\mathrm{KCl}, \mathrm{K}-\mathrm{Na}$ tartrate, and $\mathrm{KBr}$; description of the syntheses of CdSe-based core/shell QDs and their assembly into mixed crystals; images of mixed crystals embedded in silicone; description of the synthesis of reference samples used for the photostability tests. This material is available free of charge via the Internet at http:// pubs.acs.org.

\section{AUTHOR INFORMATION}

\section{Corresponding Author}

*E-mail: nikolai.gaponik@chemie.tu-dresden.de.

\section{Notes}

The authors declare no competing financial interest.

\section{ACKNOWLEDGMENTS}

Financial support from the EU FP7 Network of Excellence "Nanophotonics for Energy Efficiency" (N4E) and the BMBFTUBITAK EEEAG TUR09/001-T112E183 bilateral project is greatly acknowledged. H.V.D. also gratefully acknowledges support from NRF-CRP-6-2010-02, NRF-RF-2009-09, and ESF-EURYI. We are thankful to Prof. D. V. Talapin and to Dr. S. G. Hickey for helpful discussions. We gratefully acknowledge Christine Mickel (IFW Dresden e.V.) for assistance in performing the TEM imaging. Dr. Christian Waurisch is acknowledged for assistance in the synthesis of $\mathrm{CdSe} / \mathrm{ZnS}$ QDs.

\section{REFERENCES}

(1) Talapin, D. V.; Lee, J.-S.; Kovalenko, M. V.; Shevchenko, E. V. Prospects of Colloidal Nanocrystals for Electronic and Optoelectronic Applications. Chem. Rev. 2010, 110 (1), 389-458.
(2) Colvin, V. L.; Schlamp, M. C.; Alivisatos, A. P. Light-emitting diodes made from cadmium selenide nanocrystals and a semiconducting polymer. Nature 1994, 370 (6488), 354-7.

(3) Dabbousi, B. O.; Bawendi, M. G.; Onitsuka, O.; Rubner, M. F. Electroluminescence from CdSe quantum-dot/polymer composites. Appl. Phys. Lett. 1995, 66 (11), 1316-18.

(4) Coe, S.; Woo, W.-K.; Bawendi, M.; Bulovic, V. Electroluminescence from single monolayers of nanocrystals in molecular organic devices. Nature 2002, 420 (6917), 800-803.

(5) Gao, M.; Lesser, C.; Kirstein, S.; Möhwald, H.; Rogach, A. L.; Weller, H. Electroluminescence of different colors from polycation/ CdTe nanocrystal self-assembled films. J. Appl. Phys. 2000, 87 (5), 2297-2302.

(6) Wood, V.; Panzer, M. J.; Halpert, J. E.; Caruge, J. M.; Bawendi, M. G.; Bulovic, V. Selection of Metal Oxide Charge Transport Layers for Colloidal Quantum Dot LEDs. ACS Nano 2009, 3 (11), 35813586.

(7) Anikeeva, P. O.; Halpert, J. E.; Bawendi, M. G.; Bulovic, V. Electroluminescence from a Mixed Red-Green-Blue Colloidal Quantum Dot Monolayer. Nano Lett. 2007, 7 (8), 2196-2200.

(8) Dorn, A.; Huang, H.; Bawendi, M. G. Electroluminescence from Nanocrystals in an Electromigrated Gap Composed of Two Different Metals. Nano Lett. 2008, 8 (5), 1347-1351.

(9) Rogach, A. L.; Gaponik, N.; Lupton, J. M.; Bertoni, C.; Gallardo, D. E.; Dunn, S.; Li Pira, N.; Paderi, M.; Repetto, P.; Romanov, S. G.; O'Dwyer, C.; Sotomayor Torres, C. M.; Eychmüller, A. Light-emitting diodes with semiconductor nanocrystals. Angew. Chem., Int. Ed. 2008, 47 (35), 6538-6549.

(10) Bakueva, L.; Musikhin, S.; Hines, M. A.; Chang, T. W. F.; Tzolov, M.; Scholes, G. D.; Sargent, E. H. Size-tunable infrared (1000-1600 nm) electroluminescence from $\mathrm{PbS}$ quantum-dot nanocrystals in a semiconducting polymer. Appl. Phys. Lett. 2003, 82 (17), 2895-2897.

(11) Steckel, J. S.; Coe-Sullivan, S.; Bulovic, V.; Bawendi, M. G. 1.3 $\mathrm{mm}$ to $1.55 \mathrm{~mm}$ tunable electroluminescence from $\mathrm{PbSe}$ quantum dots embedded within an organic device. Adv. Mater. 2003, 15 (21), 18621866.

(12) Anikeeva, P. O.; Halpert, J. E.; Bawendi, M. G.; Bulovic, V. Quantum Dot Light-Emitting Devices with Electroluminescence Tunable over the Entire Visible Spectrum. Nano Lett. 2009, 9 (7), 2532-2536.

(13) Wood, V.; Halpert, J. E.; Panzer, M. J.; Bawendi, M. G.; Bulovic, V. Alternating Current Driven Electroluminescence from $\mathrm{ZnSe} /$ ZnS:Mn/ZnS Nanocrystals. Nano Lett. 2009, 9 (6), 2367-2371.

(14) Erdem, T.; Nizamoglu, S.; Sun, X. W.; Demir, H. V. A photometric investigation of ultra-efficient LEDs with high color rendering index and high luminous efficacy employing nanocrystal quantum dot luminophores. Opt. Express 2010, 18 (1), 340-347.

(15) Demir, H. V.; Nizamoglu, S.; Erdem, T.; Mutlugun, E.; Gaponik, N.; Eychmüller, A. Quantum dot integrated LEDs using photonic and excitonic color conversion. Nano Today 2011, 6, 632-647.

(16) Bendall, J. S.; Paderi, M.; Ghigliotti, F.; Li Pira, N.; Lambertini, V.; Lesnyak, V.; Gaponik, N.; Visimberga, G.; Eychmüller, A.; Sotomayor Torres, C. M.; Welland, M. E.; Gieck, C.; Marchese, L. Layer-by-Layer All-Inorganic Quantum-Dot-Based LEDs: A Simple Procedure with Robust Performance. Adv. Funct. Mater. 2010, 20, 3298-3302.

(17) Tsao, J. Y. Solid-state lighting: lamps, chips, and materials for tomorrow. IEEE Circuits Dev. Mag. 2004, 20 (3), 28-37.

(18) Dai, Q.; Duty, C. E.; Hu, M. Z. Semiconductor-NanocrystalsBased White Light-Emitting Diodes. Small 2010, 6 (15), 1577-1588.

(19) Lee, J.; Sundar, V. C.; Heine, J. R.; Bawendi, M. G.; Jensen, K. F. Full color emission from II-VI semiconductor quantum dot-polymer composites. Adv. Mater. 2000, 12 (15), 1102-1105.

(20) Jang, E.; Jun, S.; Jang, H.; Lim, J.; Kim, B.; Kim, Y. White-LightEmitting Diodes with Quantum Dot Color Converters for Display Backlights. Adv. Mater. 2010, 22 (28), 3076-3080.

(21) Erdem, T.; Demir, H. V. Semiconductor nanocrystals as rareearth alternatives. Nat. Photon. 2011, 5 (3), 126-126. 
(22) Ziegler, J.; Xu, S.; Kucur, E.; Meister, F.; Batentschuk, M.; Gindele, F.; Nann, T. Silica-Coated InP/ZnS Nanocrystals as Converter Material in White LEDs. Adv. Mater. 2008, 20 (21), $4068-4073$.

(23) Boudine, B.; Sebais, M.; Halimi, O.; Mouras, R.; Boudrioua, A.; Bourson, P. Characterization of CdS nanocrystals embedded in $\mathrm{KCl}$ single crystal matrix grown by Czochralski method. Opt. Mater. 2004, 25 (4), 373-377.

(24) Grabolle, M.; Spieles, M.; Lesnyak, V.; Gaponik, N.; Eychmüller, A.; Resch-Genger, U. Determination of the Fluorescence Quantum Yield of Quantum Dots: Suitable Procedures and Achievable Uncertainties. Anal. Chem. 2009, 81 (15), 6285-6294.

(25) Rogach, A. L.; Franzl, T.; Klar, T. A.; Feldmann, J.; Gaponik, N.; Lesnyak, V.; Shavel, A.; Eychmüller, A.; Rakovich, Y. P.; Donegan, J. F. Aqueous Synthesis of Thiol-Capped CdTe Nanocrystals: State-of-theArt. J. Phys. Chem. C 2007, 111 (40), 14628-14637.

(26) Lide, D. R. Handbook of Chemistry and Physics, 89th ed.; CRC Press, Inc.: Boca Raton, 2008-2009.

(27) Dhanaraj, G.; Byrappa, K.; Prasad, V.; Dudley, M. Handbook of Crystal Growth, 1st ed.; Springer: New York, 2010; p 1818.

(28) Holden, A.; Morrison, P. Crystals and crystal growing; MIT Press: Cambridge, MA, 1982.

(29) Wilke, K.-T.; Bohm, J. Kristallzüchtung (in German); Harri Deutsch Verlag: Frankfurt am Main, 1988.

(30) Gaponik, N.; Rogach, A. L. Thiol-capped CdTe nanocrystals: progress and perspectives of the related research fields. Phys. Chem. Chem. Phys. 2010, 12 (31), 8685-8693.

(31) Li, Y.; Jing, L.; Qiao, R.; Gao, M. Aqueous synthesis of CdTe nanocrystals: progresses and perspectives. Chem. Commun. 2011, 47 (33), 9293-9311.

(32) Donega, C. d. M.; Liljeroth, P.; Vanmaekelbergh, D. Physicochemical evaluation of the hot-injection method, a synthesis route for monodisperse nanocrystals. Small 2005, 1 (12), 1152-1162.

(33) Mayilo, S.; Hilhorst, J.; Susha, A. S.; Hoehl, C.; Franzl, T.; Klar, T. A.; Rogach, A. L.; Feldmann, J. Energy Transfer in Solution-Based Clusters of CdTe Nanocrystals Electrostatically Bound by Calcium Ions. J. Phys. Chem. C 2008, 112 (37), 14589-14594.

(34) Lesnyak, V.; Voitekhovich, S. V.; Gaponik, P. N.; Gaponik, N.; Eychmüller, A. CdTe Nanocrystals Capped with a Tetrazolyl Analogue of Thioglycolic Acid: Aqueous Synthesis, Characterization, and MetalAssisted Assembly. ACS Nano 2010, 4 (7), 4090-4096.

(35) Yang, Y.; Shi, H.-Q.; Li, W.-N.; Xiao, H.-M.; Luo, Y.-S.; Fu, S.Y.; Liu, T. Tunable photo-luminescent properties of novel transparent $\mathrm{CdSe}-\mathrm{QD} /$ silicone nanocomposites. Compos. Sci. Technol. 2011, 71 (14), 1652-1658. 